\title{
Reduced vibration and Model validation of DFIG for wind turbine
}

\author{
Wu Faming ${ }^{1 \cdot 2}$, Yang Congxin ${ }^{1}$, Yangliu ${ }^{2}$ and Wang Qing ${ }^{1}$ \\ ${ }^{1}$ College of Energy and Power Engineering, Lanzhou University of Technology, Lanzhou, 730050 \\ ${ }^{2}$ CRRC ZHUZHOUINSTITUTECO.,LTD, ZHUZHOU, 412000
}

\begin{abstract}
The vibration system of wind turbine doubly-fed induction generator (DFIG) includes generator, elastic support and frame. The work in this paper was conceived to test whether the elastic support change could change the vibration severity. So establish the Simpack simulation model and modified theoretical model to research the vibration of 4 elastic support and 5 kinds rotor mass unbalanced condition. At the same time, the experiment was carried out on the drive train test rig, experimental results are basically consistent to simulation results, and it is proved that the research in the paper is correct. Meanwhile, it proved that modified theoretical analysis model can be use to vibration analysis in frequency domain and the Simpack simulation model can be use to vibration analysis in time domain(such as trend analysis) for the rotor unbalance.
\end{abstract}

\section{Introduction}

Wind power is the world's fastest growing renewable energy source. With the rapid development of wind power in recent years, the capacity of wind turbine becomes larger and larger, the blade is longer and the tower is getting higher and higher, the characteristics of the flexibility are more obvious ${ }^{[1]}$. Therefore, the vibration problem of wind turbine also becomes more and more obvious and becomes the focus of research. Moreover, vibration is an important parameter to reflect the condition of wind turbines, and serious vibration is also one of the important reasons for damage wind turbine. Therefore, the research serious vibration in the operation of wind turbines is of great significance for the safe operation and the prolonging of its service life. However, the vibration problem of the high speed side generator system of wind turbines is more serious and becomes one of the main sources of vibration ${ }^{[2-3]}$.

To the authors' knowledge, there have been no similar studies reported in the literature, thus the analysis in this article may open a new window for extension and more research. The vibration of DFIG is a complex problem, which is affected by the characteristics of the generator itself, elastic support, frame and operation characteristics ${ }^{[4-6]}$. Based on the structure and operation characteristics of the wind turbine, the frequency spectrum analysis and time domain analysis are applied to the design of the DFIG. Through the Simpack simulation model, the sensitivity of the rotor unbalance and elastic installation of DFIG fault is studied, and the modified theoretical model is verified by the vibration test of the DFIG. Through research and analysis, the theoretical model of vibration analysis for DFIG are proposed, providing an efficient method for selecting elastic support.

* Corresponding author: wufm@csrzic.com

\section{Research method and simulation model}

In order to study the vibration problem of DFIG. First of all, it is necessary to clear the vibration evaluation criteria, which is widely accepted to judge the vibration of rotating machinery: ISO 10816 of international standardization organization ${ }^{[7]}$, VDI 3834 in Germany ${ }^{[8]}$, and $\mathrm{GB} / \mathrm{T} 10068$ in $\mathrm{China}^{[9]}$. In addition, the excitation of DFIG vibration system caused by rotor imbalance should be focused on, and modified theoretical model of vibration analysis for DFIG is also discussed.

\subsection{Vibration evaluation criteria}

Vibration evaluation criteria look up the standard and Table1 shows the classification of vibration severity zones and value : ISO 10816-3"Mechanical vibrationEvaluation of machine vibration by measurements on non-rotating parts- Part3" points out, Large machines with rated power above $300 \mathrm{~kW}$ and not more than 50 MW, Electrical machines with shaft height $\mathrm{H} \geq 315 \mathrm{~mm}$; National standard GB10068 "Mechanical vibration of certain machines with shaft heights $56 \mathrm{~mm}$ and higherMeasurement, evaluation and limits of vibration"; VDI 3834 "Measurement and evaluation of the mechanical vibration of wind energy turbines and their components", the vibration of the wind turbine were obtained from a statistical analysis of the measured vibration values from more than 450 wind turbine. 
Table 1. Classification of vibration severity zones for machines(r.m.s Velocity $\mathrm{mm} / \mathrm{s}$ )

\begin{tabular}{|c|c|c|c|}
\hline zones & ISO 10816 & GB10068 & VDI 3834 \\
\hline A & 3.5 & 3.5 & 6 \\
\hline B & 7.1 & $/$ & 10 \\
\hline $\begin{array}{l}\text { A: Machines with vibration within this zone are normally } \\
\text { considered acceptable for unrestricted long term operation. }\end{array}$ \\
B: Machines with vibration within this zone are normally \\
considered unsatisfactory for long-term continuous \\
operation. Generally, the machine may be operated for a \\
limited period in this condition until a suitable opportunity \\
arises for remedial action.
\end{tabular}

\subsection{Rotor mass unbalance}

It is known that the rotating part (such as the generator rotor) has the unbalanced mass, the radial force will be produced during the operation.

$$
F=m \cdot e \cdot\left(\frac{\pi \cdot n}{30}\right)^{2}
$$

Where: $m$-unbalance mass; $e$-Distance between unbalance and the axis of rotation of the object; $n-$ rotating speed.

It can be seen from the formula that the size of the radial centrifugal force is proportional to the square of the rotating speed. If the rotor mass unbalance exceeds the allowable limit, it will cause an excessive vibration which may lead to machine failure. Therefore, the regular rotor balancing is often required to keep machine vibration within acceptable level.

\subsection{Theoretical model of vibration analysis for the DFIG}

Although the high development of computer and finite element software have facilitated the natural frequency computation of very complicated structures, the simplified algorithms are still applied to estimating the Natural Frequency of Multi-degree Freedom Vibration System $^{[10]}$. The DFIG system includes generator, elastic support and frame is similar to the Multi-degree Freedom Vibration System. Therefore, this method can be used to establish the theoretical model of vibration analysis for DFIG. The calculation formula is as follows:

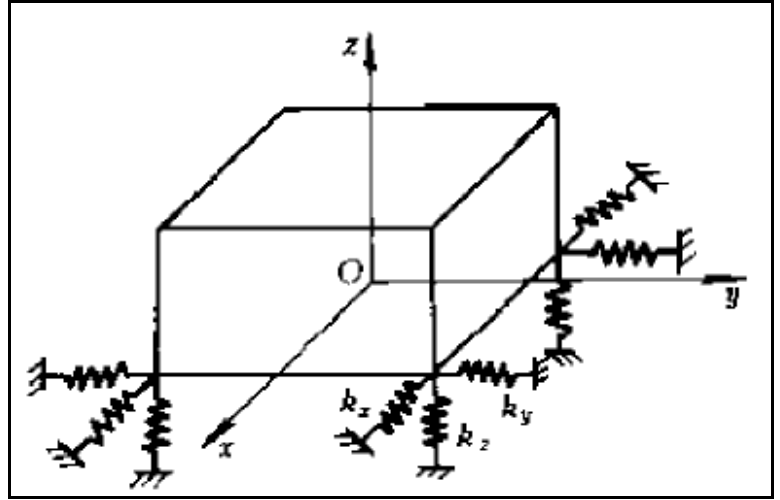

Fig.1. Theoretical model of vibration analysis for DFIG
Model for $\phi_{z}$ :

$\omega_{\phi_{z}}=\frac{k_{y} \sum a_{i}^{2}+k_{x} \sum b_{i}^{2}}{I_{z}}$

Model for $\phi_{y}$ :

$\omega^{2}=\frac{1}{2}\left[\left(\omega_{x}^{2}+\omega_{\phi_{y}}^{2}\right)+\sqrt{\left(\omega_{x}^{2}-\omega_{\phi_{y}}^{2}\right)^{2}+\frac{4 \omega_{x}^{4} h^{2} m}{I_{y}}}\right]$

Model for $\phi_{x}$ :

$\omega^{2}=\frac{1}{2}\left[\left(\omega_{y}^{2}+\omega_{\phi_{x}}^{2}\right)+\sqrt{\left(\omega_{y}^{2}-\omega_{\phi_{x}}^{2}\right)^{2}+\frac{4 \omega_{y}^{4} h^{2} m}{I_{x}}}\right]$

$f=\frac{\omega}{2 \pi}$

where:

$$
\begin{aligned}
& \omega_{x}^{2}=k_{x} / m ; \quad \omega_{\phi_{y}}^{2}=\left[k_{x} \sum a_{i}^{2}+k_{x} h^{2}\right] / I_{y} \\
& \omega_{y}^{2}=k_{y} / m ; \quad \omega_{\phi_{x}}^{2}=\left[k_{z} \sum a_{i}^{2}+k_{y} h^{2}\right] / I_{x} \\
& \quad k_{x}, \quad k_{y}, k_{z}-\mathrm{x} 、 \mathrm{y} 、 \mathrm{z} \text { Rigidity; } \\
& I_{x}, \quad I_{y}, \quad I_{z}-\text { Rotational inertia of generator to x, }
\end{aligned}
$$
$\mathrm{y}, \mathrm{z}$ axis;

$a_{i}, b_{i}, h-$ The coordinates of the center points at the connection between the isolators and the equipment;

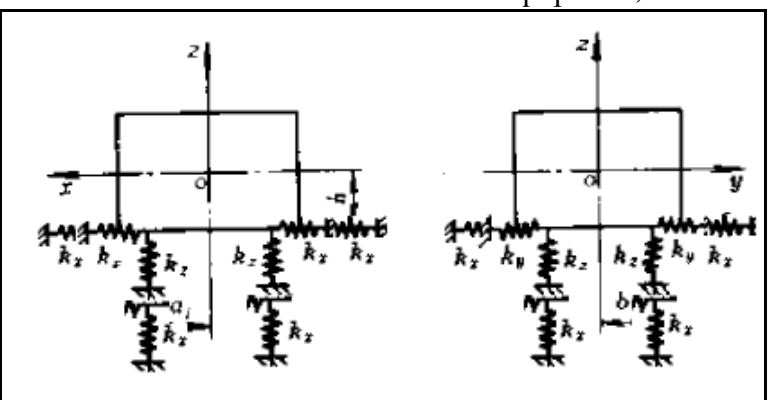

Fig. 2. Modified theoretical model of vibration analysis for DFIG

The vibration system of generator has its particularity. The flexibility of the frame affects the system frequency. Therefore, the multi free vibration isolation system needs to be modified. The modified theoretical model of the vibration system is shown in Fig.2. Modified the stiffness of three directions of $\mathrm{x}, \mathrm{y}$ and $\mathrm{z}$ from the frame. $k_{x}=\frac{k_{x 1} \cdot k_{x 2}}{k_{x 1}+k_{x 2}}, \quad k_{y}=\frac{k_{y 1} \cdot k_{y 2}}{k_{y 1}+k_{y 2}}, \quad k_{x}=\frac{k_{z 1} \cdot k_{z 2}}{k_{z 1}+k_{z 2}}$

\subsection{Simpack simulation model of vibration analysis for DFIG}

In order to realistically reappear the actual operate state, establishing the Simpack simulation model of vibration analysis for DFIG, which is mainly composed of stator insert, rotor core, rotating shaft, frame and elastic support. The 3D model of the system is shown in Figure 3. The 4 elastic supports are simplified as springs, and $X$, $\mathrm{Y}$ and $\mathrm{Z}$ are applied to simulate the supporting effect in 3 
directions. The frame is built into a flexible body. The dynamic model can be used for modal analysis, rotor unbalance analysis, sweep frequency analysis and vibration amplitude analysis.

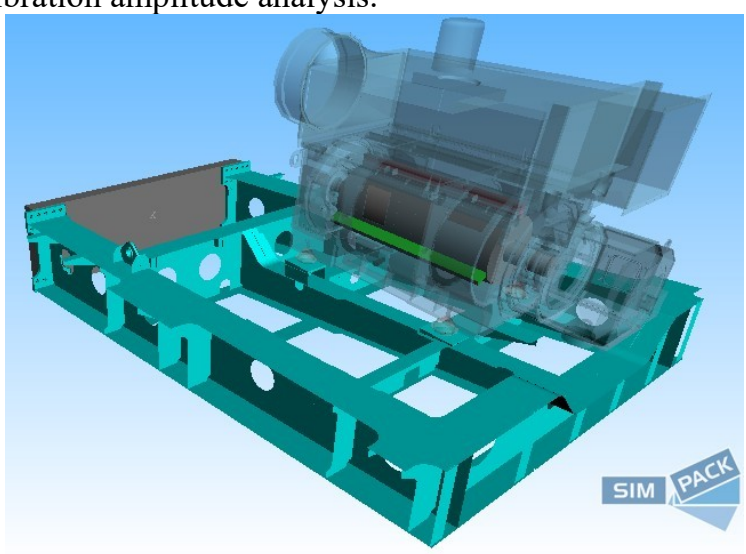

Fig.3. 3D model for vibration mode of the DFIG

\section{Analysis model and simulation results}

In order to research the influence of elastic support on Simpack simulation model and theoretical model, 4 different elastic support have been analyzed. The values of stiffness parameter of elastic support are shown in Table2. The values of the generator parameter are shown in Table3.

Table 2. Stiffness parameter of the elastic support

\begin{tabular}{|c|c|c|c|c|}
\hline Stiffness & A & B & C & D \\
\hline $\mathrm{k}_{\mathrm{x}}(\mathrm{kN} / \mathrm{mm})$ & 10 & 10 & 12 & 15 \\
\hline $\mathrm{k}_{\mathrm{y}}(\mathrm{kN} / \mathrm{mm})$ & 10 & 10 & 12 & 15 \\
\hline $\mathrm{k}_{\mathrm{z}}(\mathrm{kN} / \mathrm{mm})$ & 12 & 30 & 35 & 35 \\
\hline
\end{tabular}

Table 3. Parameter of Generator

\begin{tabular}{|c|c|c|}
\hline Parameter & Value & unit \\
\hline Rate power & 2000 & $\mathrm{~kW}$ \\
\hline mass & 7800 & $\mathrm{Kg}$ \\
\hline Rate speed & 1680 & $\mathrm{rpm}$ \\
\hline Rev range & $1000 \sim 1680$ & $\mathrm{rpm}$ \\
\hline Ix & 1868 & $\mathrm{Kg} \cdot \mathrm{m}^{2}$ \\
\hline Iy & 3322 & $\mathrm{Kg} \cdot \mathrm{m}^{2}$ \\
\hline $\mathrm{Iz}$ & 2662 & $\mathrm{Kg} \cdot \mathrm{m}^{2}$ \\
\hline
\end{tabular}

\subsection{Vibration analysis in frequency domain of theoretical model}

Both theoretical model and modified theoretical model of vibration analysis are used to calculate the natural frequency of 4 kinds elastic support. The results are shown in Table 4 and Table5.

Table 4. Relationship between stiffness and natural frequency of elastic support (theoretical model)

\begin{tabular}{|c|c|c|c|c|}
\hline $\begin{array}{c}\text { Torsional } \\
\text { frequency }\end{array}$ & A & B & C & D \\
\hline$\emptyset_{\mathrm{x}}$ & 29.7 & 44.5 & 48.1 & 54.5 \\
\hline
\end{tabular}

\begin{tabular}{|c|c|c|c|c|}
\hline$\emptyset_{\mathrm{y}}$ & 25.2 & 36.7 & 39.7 & 44.9 \\
\hline$\emptyset_{\mathrm{z}}$ & 21.4 & 33.8 & 36.5 & 41.4 \\
\hline
\end{tabular}

From the above results, it can be seen that the calculated by the modified theoretical model are obviously lower, which are basically the same as the test result. The reason is that the modified theoretical model considered the flexibility of the frame. On the other hand, with the increase of stiffness, the torsional natural frequency of the three directions of DGIF increases to a certain extent, and the ratio of the frequency rise is about $25 \% \sim 30 \%$ on the stiffness, which can provide advice for the design and selection of elastic support.

Table 5. Relationship between stiffness and natural frequency of elastic support (modified theoretical model)

\begin{tabular}{|c|c|c|c|c|}
\hline $\begin{array}{c}\text { Torsional } \\
\text { direction }\end{array}$ & $\mathrm{A}$ & $\mathrm{B}$ & $\mathrm{C}$ & $\mathrm{D}$ \\
\hline$\emptyset_{\mathrm{x}}$ & 24.5 & 30.1 & 31.2 & 32.7 \\
\hline$\emptyset_{\mathrm{y}}$ & 21.1 & 27.4 & 28.6 & 30.4 \\
\hline$\emptyset_{\mathrm{z}}$ & 18.4 & 24 & 24.9 & 26.4 \\
\hline
\end{tabular}

\subsection{Vibration analysis in frequency domain of Simpack simulation model}

Similarly, the Simpack simulation model is used to calculate the natural frequency. The results are shown in Table 6:

Table 6. Relationship between stiffness and natural frequency of elastic support ( Simpack model)

\begin{tabular}{|c|c|c|c|c|}
\hline $\begin{array}{c}\text { Torsional } \\
\text { frequency }\end{array}$ & A & B & C & D \\
\hline$\emptyset_{\mathrm{x}}$ & 13.9 & 14.4 & 15.5 & 17.0 \\
\hline$\emptyset_{\mathrm{y}}$ & 17.9 & 22.8 & 24.3 & 26.3 \\
\hline$\emptyset_{\mathrm{z}}$ & 16.9 & 23.7 & 24.7 & 26.6 \\
\hline
\end{tabular}

From the above results, we can see that the results calculated by Simpack model are close to the modified theoretical model. As shown in Table 6, the deviation of the $\emptyset_{\mathrm{z}}$ direction is about $5 \%, \emptyset_{\mathrm{x}}$ and $\emptyset_{\mathrm{y}}$ is about $50 \%$, $15 \%$. Similarly, with the rise of the stiffness, the torsional frequency of the $\emptyset_{\mathrm{z}}$ all increase to a certain extent, and the ratio of the frequency rise is about $35 \%$ of the stiffness. But the torsional frequency of $\varnothing x$ and $\emptyset y$ varies little with the stiffness, and looks at the Simpack $\mathrm{X}$ axis, and the $\mathrm{Y}$ axis finds that the reason for the two directions of the generator system mode and the frame mode fusion. Therefore, the modified theoretical model needs to introduce the torsion correction factor $\mathrm{T} 1=15.7$ for $\emptyset \mathrm{x}$ and $\mathrm{T} 2=4$ for $\emptyset \mathrm{y}$. The revised results are as table 7 . From the result, the percentage deviation will reach 5\% after increasing the frequency correction coefficient.

Table 7. Comparison of modal between Simpack model and modified theoretical model

\begin{tabular}{|c|c|c|c|c|}
\hline $\begin{array}{c}\text { Torsional } \\
\text { frequency }\end{array}$ & A & B & C & D \\
\hline$\varnothing \mathrm{x}$ & $-36.69 \%$ & $0.26 \%$ & $0.22 \%$ & $-0.02 \%$ \\
\hline$\varnothing \mathrm{y}$ & $-4.65 \%$ & $2.76 \%$ & $1.33 \%$ & $0.36 \%$ \\
\hline
\end{tabular}


$\emptyset_{\mathrm{z}}$ $9.04 \%$

$1.24 \%$

$0.69 \%$

$-0.79 \%$

\subsection{Vibration analysis in time domain of Simpack simulation model}

Rotor unbalance is the most common fault in various rotating machines. According to statistics, nearly $70 \%$ of the faults in rotating machinery are related to rotor unbalance. Unbalance of generator rotors is a major contributor to generator downtime, costing power producers hundreds of thousands of dollars in lost revenue each year. proper attention to design detail, manufacturing tolerances and procedures, during initial manufacture or subsequent rewinds, can minimize the possibility of vibration related incidents. Rotor unbalance has many causes.

This paper focus on study that the relationship between the rotor mass unbalance and the vibration severity. By changing the torque input of the front of the drive train model to change the generator speed $1000 \sim 1700 \mathrm{rpm}(16.7 \sim 28.3 \mathrm{~Hz})$, and the rotor mass unbalance $e$ is $0.02 \mathrm{~mm}, 0.06 \mathrm{~mm}, 0.10 \mathrm{~mm}, 0.14 \mathrm{~mm}$, $0.18 \mathrm{~mm}, 0.22 \mathrm{~mm}$, and B elastic support are carried out. The simulation of frequency sweep is carried out in the interval. The simulation result is shown in Fig4 and Fig5.

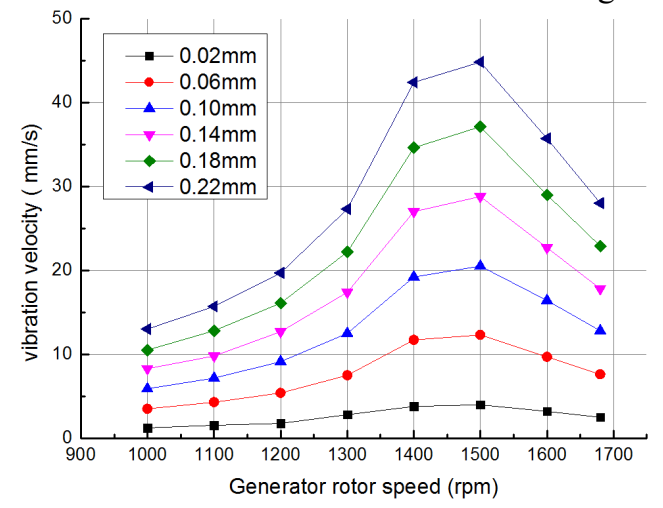

Fig.4. Relationship between Amplitude of vibration velocity and Generator speed $\left(\emptyset_{\mathrm{z}}\right)$

Fig.4 is the simulation results of the rotor system $\emptyset_{\mathrm{z}}$ vibration velocity with different rotor mass unbalance. it can be seen that vibration severity increases with the increase of the rotor unbalance. so we should control the rotor unbalance to reduce the vibration severity.

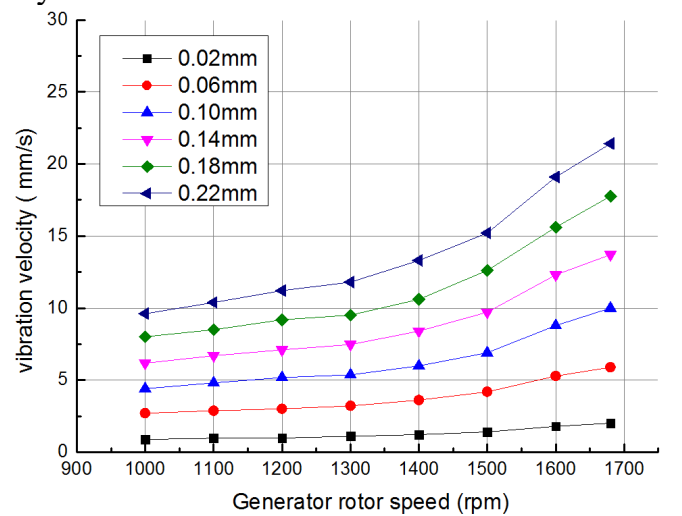

Fig.5. Relationship between Amplitude of vibration velocity and Generator speed $\left(\emptyset_{\mathrm{y}}\right)$
Fig. 5 is the simulation results of the rotor system $\emptyset_{\mathrm{y}}$ vibration velocity with different rotor mass unbalance. it can be seen that vibration severity increases with the increase of the rotor unbalance. So we should control the rotor unbalance to reduce the vibration severity.

From Fig.4 and Fig.5, we found that the Amplitude of vibration velocity is increasing with generator speed increasing. but the Amplitude of vibration velocity of the $\emptyset_{z}$ is increasing with generator speed increasing until $1450 \mathrm{rpm}$, the value is down. The main reason is that the damping value of the $\emptyset_{\mathrm{z}}$ direction is small, and it is more easily excited. The $\emptyset_{\mathrm{y}}$ direction has frame support, and its damping is large. Even resonance occurs, but it is not cause the resonance. Judging from the vibration severity amplitude, the case that rotor unbalance under $0.02 \mathrm{~mm}$ is the best choice for vibration suppression, which meets the vibration standard and the user's requirement.

\section{Experimental verification}

\subsection{Experiment setup}

The experiments were carried out on the drive train test rig, as shown in Fig.6. Stiffness of the elastic support is chose the type B.

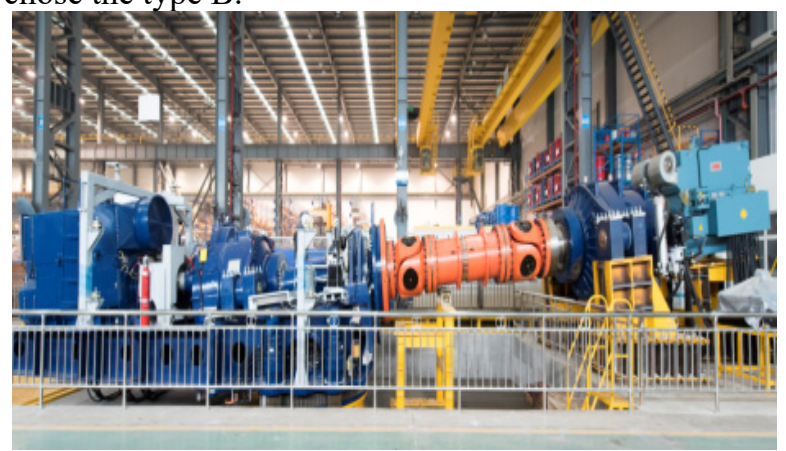

Fig.6. Whole test rig

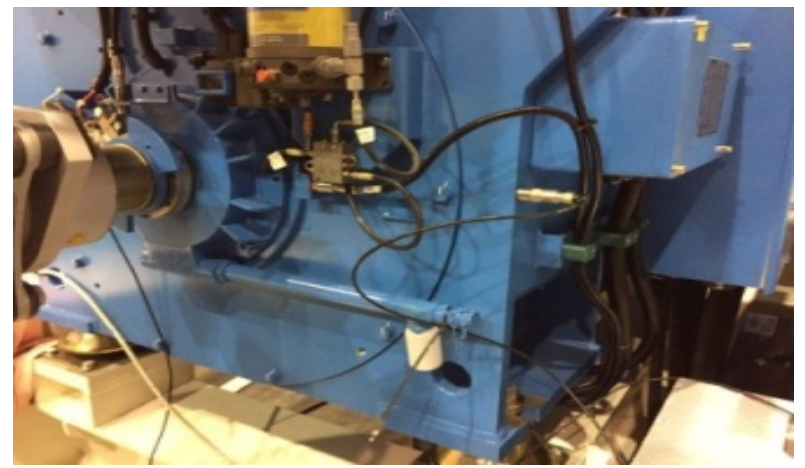

Fig.7. Physical sensor installation

Four accelerometers were attached inside the DFIG, three accelerometers use to test the generator and one accelerometers use to test the gearbox high speed shaft. vibration data was collected while the machine was in operation.

Table 8. Sensor notations and descriptions

\begin{tabular}{|c|c|}
\hline Sensor Label & Description \\
\hline GB_X & Gearbox high speed shaft axial \\
\hline
\end{tabular}




\begin{tabular}{|c|c|}
\hline GE_X & Generator drive end axial \\
\hline GE_Y & Generator drive end radial(V) \\
\hline GE Z & Generator drive end radial(H) \\
\hline
\end{tabular}

The mounting locations were chosen to reflect typical sensor placement practices seen in commercial wind turbine drive train vibration-based condition monitoring systems. The physical installation of these accelerometers is shown in Fig.7, with sensor labels and descriptions given in Table 8 .

\subsection{Parameters effect validation}

According to the effective value statistics, the vibration acceleration of the generator in the three direction is all rising with the speed fluctuation, but it is worth noting that at $1440 \mathrm{rpm}$, the transverse speed of the generator falls down, and the vertical speed has a wave peak. It is basically consistent with the Simpack simulation results, proving the simulation model.

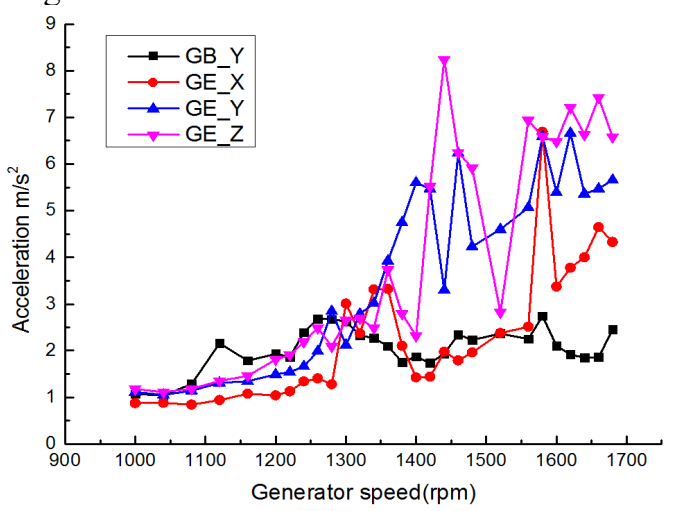

Fig.8. Relationship between Amplitude of Rotational acceleration and Generator speed

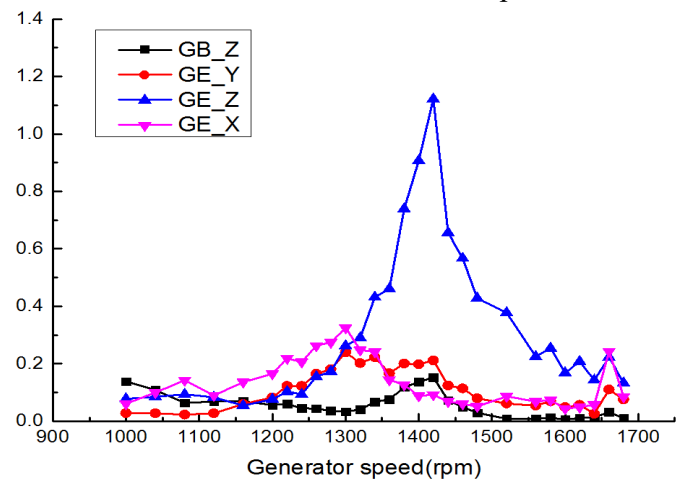

Fig.9. Relationship between Amplitude of Rotational Frequency and Generator speed

The frequency analysis is used to extract the frequency component, and the statistics are used to get the following figure 9. From the trend diagram, it can be clearly seen that the vibration of the generator reaches a peak at 1420rpm, and its amplitude is far greater than other directions, and the acceleration in other directions does not rise and reverse at this speed. Also, It is basically consistent with the Simpack simulation results, proving the simulation model.

Comparing the experimental results with the simulation results. although there are still some differences between the experimental and the simulation results, the main principles of the model can be verified.

\section{Conclusions}

This paper establish the Simpack simulation model and modified theoretical model to research the vibration of DFIG. The principles and fundamental characteristics of the vibration analysis of DFIG were studied analytically and experimentally, some conclusions are as follows:

1) The proposed modified theoretical model is effective. The vibration system of DFIG whose resonant frequency can be changed by turning the stiffness of elastic support.

2) The proposed simpack simulation model is effective. The natural frequency around $\mathrm{z}$ of DFIG should avoid being in the range speed, so as to avoid resonance, which can reduce the vibration severity.

3) It is found that the rotor unbalance increase influence the stability of DFIG during period on operation.

\section{Acknowledgment}

The current thesis was supported by National Basic Research Program of China/ 973 Project(2014C B046201) and the National Science Foundation of China ( 11262011).

\section{References}

1. Masoud Asgarpour. Bayesian Based Diagnostic Model for Condition Based Maintenance of Offshore Wind Farms[J]. Energies , 2018, 11 (2) :300

2. E Mollasalehi. Indicative Fault Diagnosis of Wind Turbine Generator Bearings Using Tower Sound and Vibration[J]. Energies , 2017,10 (11):1853

3. Tony Burton, David Sharpe, Nick Jenkins. Wind Energy Handbook[M]. First published: 26 April 2002

4. P Zhao, X Li, J Yang, L Yang .Theoretical and Experimental Research on Vibration Characteristics of the Doubly-Fed Induction Generator for Wind Turbine[J]. International Conference on Cybernetics , 2016 :60-64

5. Y Gao,H Dai , X Li. Application Analysis of Vibration for High Speed Shaft Bearings Used in Wind Turbine Gearbox[J]. Bearing , 2016

6. Radosław Zimroz, J Urbanek, Tomasz Barszcz . Measurement of Instantaneous Shaft Speed by Advanced Vibration Signal Processing - Application to Wind Turbine Gearbox[J]. Metrology \& Measurement Systems , 2011 , 18 (4) :701-712

7. ISO 10816-3:2009 ,Mechanical vibrationEvaluation of machine vibration by measurements on non-rotating parts- Part3[S].

8. VDI 3834-1:2009, Measurement and Evaluation of the Mechanical Vibration of Wind Energy Turbines and Their Components-Onshore Wind Energy Turbines With Gears [S]. 
9. GB10068-2008, Mechanical vibration of certain machines with shaft heights $56 \mathrm{~mm}$ and higherMeasurement, evaluation and limits of vibration[S].

10. Weide Qu, Hengling Tang. Mechanical vibration Manual 2nd edition[M]. Machinery Industry Press, 2005,5 